\title{
Il volto di Dio, il volto di Laura. Appunti preliminari ${ }^{1}$
}

\section{Giorgio Bertone}

Università di Genova

\begin{abstract}
L'Autore esamina Rvf 16, 77, 78 (chiarendone fino in fondo l'esegesi minuta) sostanzialmente per approfondire i rapporti di P. con l'Immagine e con il Ritratto (quello di Laura eseguito da Simone Martini, e l'idea di ritratto che emerge dai sonetti gemellati). L'esame e condotto sia sul retroterra storico-teologico implicato nel sonetto del romeo, sia sul nuovo statuto che l'immagine eseguita da un artista laico di un volto laico (nel dipinto di Simone) acquista dentro il percorso del Canzoniere, non coincidente - e questa è un'ulteriore tesi del saggio che si discosta dagli studi recenti, anche di storici dell'arte- con ció che $\mathrm{P}$. asserisce in altre opere ove tratta delle arti liberali. La nascita del ritratto moderno risulta infine inscindibile dalla rappresentazione antica e più recente del volto divino.
\end{abstract}

Parole chiavi: Petrarca, Rvf 16, 77, 78, dalla Vera Icon al Ritratto.

\section{Abstract}

The author examines Rvf 16, 77, 78 (in considerable depth and detail) substantially in order to provide a fuller understanding of the relationships held by P. with Image and Portrait (that of Laura by Simone Martini, and also the notion of portrait that emerges from the conceptually related sonnets). Whether concerning the historico-theological background involved in the pilgrim's sonnet, or whether focusing on the new statute given by a lay artist to the image of a lay face (that painted by Simone) acquired within the scope and developments of the "Canzoniere», this current assessment is set forth in a manner that is non-coincident - and this is an additional thesis in this essay that differs from recent studies, even those of art historians - with that which P. asserts in other works dealing with the liberal arts. The birth of modern portrait is ultimately inseparable from both the ancient and more recent representations of the divine face.

Key words: Petrarch, Rvf 16, 77, 78, from the Vera Icon to the Portrait.

1. Il nucleo di idee di questo contributo appartiene alla data e alla sede del bel convegno barcellonese. In seguito ho continuato a sviluppare concetti, letture, riletture, —anche per le sollecitazioni conviviali di allora, soprattutto quelle dell'amico Rossend Arques, che ringrazio di cuore-, fin che mi è lievitato sotto gli occhi «mai stanchi, per mio mal», un saggio troppo lungo per essere qui ospitato. Senza rinunciare a presentare i risultati salienti di ricerca e le implicite recensioni o esame funzionale al mio scopo, dei libri più belli usciti di fresco su $R v f 16,77,78$ e sulla rete di temi che questi testi continuano a tessere, scorcio qui drasticamente il discorso, il quale risulterà dunque tagliato alla brava, qua e là lacunoso di referti, esempî, rinvii bibliografici. Necessita di un lettore volonteroso e amico. 
Che sulla scorta di Plinio il Vecchio (postillato in Par. lat. 6802, c.256 v.) Petrarca potesse facilmente dedurre un preciso accredito alla pittura di status d'arte liberale è già una notizia importante. ${ }^{2}$ Non sufficit. L'intento di cogliere l'essenza di una mossa culturale che riporta sul tavolo della discussione sempre più fitta degli esegeti di varia stirpe un repertorio terminologico di sommo impegno — «idolo», «icona», «immagine», «forma», «ritrarre»—, obbligherà a convergere ancora sui tre sonetti $(16,77,78)$ come luogo di concentrazione, sintesi e superamento. ${ }^{3}$ E pur vero che la postilla manoscritta a margine del passo della Storia naturale in cui Plinio accenna all'urbanità di Apelle e di Simone (oggi diremmo con parola frusta e degradata alla misura privata, «simpatia») ha il valore di attestato se calata in combinato disposto con i due esametri sulle glorie gemelle di Mantova e Siena del frontespizio del manoscritto Ambrosiano, «benservito del cliente soddisfatto» (Contini), ${ }^{4}$ che rappresenta «forse il più alto tributo rivolto a un artista dopo l'antichità». ${ }^{5}$ Un attestato, evidentemente, della qualità dell'arte di Simone pittore, subito colto al volo e rilanciato dal Vasari quando nelle Vite citando i due sonetti del Petrarca asserisce che il Poeta "ha dato più fama alla povera vita di mastro Simone, che non hanno fatto né faranno mai tutte l'opere sue». ${ }^{6}$ (Povero Simone, schiacciato dalla supremazia storica della Poesia!). E sarà centrale nel capitolo sui rapporti tra poesia e arti e sulla garanzia d'eternità che la prima assicura alle seconde (che è poi il Leitmotiv delle Vite vasariane); nonché nella biografica storicoartistica di Petrarca medesimo. A meno che non lo si consideri già quale aspetto più ufficiale del discorso tutto interno alla discussione, come dire, tecnica, stretta stretta tra le due arti e che si fonda, per esempio, sulla duplicità terminologica di parole come "carte» («carte», pergamene su cui scrivere, "carte», pergamene su cui dipingere, miniare; «digitus», — «digito» negli esametri"dito" per scrivere, «dito" per dipingere). ${ }^{7}$

2. Edouard Pommier, Il ritratto. Storia e teorie dal Rinascimento all'età dei Lumi, Torino: Einaudi, 2003, p. 25. Nel De tabulis pictis (De rem. 1,40) Petrarca attribuisce alla Grecia l'elevazione della pittura ad arte liberale.

3. Già Enrico FENZI, Note petrarchesche: Rvf 16, Movesi il vecchierel, in Id., Saggi petrarcheschi, Firenze: Cadmo, 2003, p. 15-40, e in chiave semiotica Marcello CicCUTO, Figure di Petrarca. Giotto, Simone Martini, Franco Bolognese, Napoli: Federico \& Ardia, 1991, hanno in diversi modi raccordato i tre sonetti. Ora si veda il pressoché monumentale commento di Rosanna Bettarini, _-in cui le note ai singoli testi costituiscono dei veri e propri saggi in forma di glossa,- - ciò che riguarda espressamente 16, 77, 78 (Francesco PETRARCA, Canzoniere Rerum Vulgarium Fragmenta, a cura di Rosanna Bettarini, Torino: Einaudi, 2005).

4. Gianfranco Contini, Petrarca e le arti liberali, in Francesco Petrarca citizen of the world, a cura di A. Bernardo, Padova - Albany, 1980, p. 115-131.

5. Maria Monica DonATO, «Veteres» e «novi», «externi» e «nostri». Gli artisti di Petrarca: per una rilettura, in Il Medioevo: immagini e racconto (Atti del Convegno Internazionale di Studi, Parma, 27-30, settembre 2000), Milano: Electa, 2003, p. 432-433. Il ben noto distico in questione: «Mantua Virgilium, qui talia carmina finxit / Sena tulit Symonem, digito qui talia pinxit».

6. Giorgio VASARI, Le Vite de più eccellenti pittori, scultori e architettori nelle redazioni del 1550 e 1568, a cura di R, Bettarini e p. Barocchi, Firenze,1967, t. II, p. 191-192.

7. V. più avanti le osservazioni sulla terminologia adibita nei sonetti. 


\section{Un possibile "autoritratto" per delega e una teoresi nascosta nei versi}

Accogliere poi l'ipotesi di Maria Monica Donato ${ }^{8}$ che nell'Allegoria che funge da frontespizio al Virgilio Ambrosiano (tra il '38 e il '43), e precisamente nella figura di Virgilio poeta laureato, si possa leggere un «ritratto indiretto» del Petrarca, - che si affiancherebbe dunque alla cooptazione di Simone e con lui della pittura al grado e valore della Poesia—, nonché la notizia che "Petrarca è il primo soggetto noto d'una diffusa ritrattistica indipendente, ciò che è tanto più notevole trattandosi d'un privato, effigiato già in vita solo per la sua fama [e spesso colto in "istantanee" non autorizzate], ed è anche il primo individuo a vantare una tradizione fisionomicamente riconoscibile, basata su disegni dal vero", non fa che acuire l'urgenza di aprire lo scrigno dei tre sonetti. Che pensava, l'interessato, del ritratto laico in sede assoluta, ovvero nella lirica? (E non ci stancheremo di rimarcare l'eccezionalità delle sede, sotto ogni punto di vista).

Dato dunque per acquisito il riesame di tutti i passi dedicati alle arti plastiche nell'opera omnia, e avanzata l'ipotesi che nel complesso vi si possano reperire gli elementi sparsi di una virtuale teoria sul ritratto già ricca di tutte le tensioni future, questa eventuale teoria anticipatrice risulterà, in quanto teoresi, marchiata di un tasso imprecisabile di involontarietà e tangibilmente frammentaria e frastagliata, persino incoerente. Circoscrivere in un ambito privilegiato i sonetti in questione non comporta dunque consegnarli alla sfera della pura teoresi in versi - e come potrebbero all'interno di un libro così poco teorico per istituzione e vocazione come il Canzoniere?-, piuttosto significa disporsi a catturare la serie fitta di elementi non solo linguistici e terminologici che come in altri casi, per esempio nella teoria della lingua poetica dove Petrarca non ci ha consegnato alcun trattato de vulgari eloquentia o similia, ma una prassi poetica che la sottintende e la ingloba, qua e là l'accenna con gesto perentorio o dissimulato - fanno emergere davanti ai nostri occhi una scelta precisa: introdurre nelle pieghe della vicenda d'amore e d'errore, anzi, intrecciare ad essa pure le novità delle acquisizioni culturali, impregnare l'arduo amore per Laura delle risultanze dell'arduo riflettere sulle idee e, qui, sull'arte. E viceversa. Non si dà desiderio senza speculazione. Meglio: la speculazione, la sintesi fulminea e complessiva di una questione complessa, millenaria, si attua qui e solo qui sotto la stretta cogenza del Desiderio. L'amore per Laura non ammette divagazioni. Né appunti in margine. Tanto più se ciò che viene innanzi è proprio la sua Imago.

\section{Rvf 16.}

E andiamo a incomiciare con i sonetti, di cui offriamo, come richiedeva Franco Fortini, una «onesta parafrasi». ${ }^{9}$ Ho preferitoo «idea» o «immagine

8. Maria Monica Donato, op. cit., p. 446-448.

9. Se ne parte il vecchierello canuto e bianco (sinonimi? bianco di pelo e pallido in volto? vecchio di età e bianco di pelo? dittologia stereotipica?) dal dolce (caro, amato) paese dove ha portato a compimento le stagioni della sua vita e (se ne parte) dalla sua famigliola sgomenta 
ideale» a «immagine» sic et simpliciter, per le ragioni che verranno dette più avanti e che attengono alla gerarchia dei modelli. "In altrui»: vale proprio "in altre donne». ${ }^{10}$ Se alleghiamo anche la storia del motivo sviluppato nei prede-

che osserva il caro padre andarsene e venire a mancare; di lì (dal suo paese e dalla sua famiglia), trascinando poi il vecchio corpo nelle ultime tappe (o stazioni) della sua esistenza, si fa forza con la buona volontà quanto più gli è possibile, malridotto (affranto) dagli anni e fiaccato dal (lungo) cammino; e giunge a Roma, perseguendo il fine desiderato che si era proposto, per ammirare l'aspetto di Colui (il volto di Cristo, la Veronica di San Pietro) che (il vecchio romeo) spera di vedere di nuovo lassù in Paradiso: in tal modo, misero me, a volte io cerco, o donna mia, per quanto ( $\mathrm{mi}$ ) è possibile, in altre donne la (tanto) desiderata vostra vera forma (la vostra immagine ideale).

10. Francesco Petrarca, Canzoniere, testo critico e introduzione di Gianfranco Contini, annotazioni di Daniele Ponchiroli, Torino: Einaudi, 1964, p. 18; Francesco Petrarca, Canzoniere, edizione commentata a cura di Marco Santagata, Milano: Mondadori, 1996, p. 70: "Vado talvolta cercando, in altre donne la vostra immagine (da me desiderata come è desiderata dal vecchierello quella della Veronica)». V. il sottile commento di Rosanna Bettarini: "Nelle altre sembianze simili e sostanze sensibili, quindi anche nelle altre donne (nel contesto suggerito dalla prima quartina del sonetto XIII), che sono "sembianza" terrena o "essemplo" della sostanza celestiale della donna o domina invocata.» (Francesco.PETRARCA., Canzoniere Rerum Vulgarium Fragmenta, a cura di Rosanna Bettarini, cit., p. 74). Enrico FEnzI, Note petrarchesche: Rvf 16, Movesi il vecchierel, cit., p. 21: «essendone al presente impedito dalla lontananza, non può fare a meno di cercarla "in altrui", cioè nelle sembianze di altre donne che in qualche modo, approssimativo, insufficiente eppur irrinunciabile, gliela restituiscano». Poco dopo, con parziale spostamento: «In altri termini, per essere chiaro sino al superfluo, Petrarca non va in cerca di altre donne che assomiglino a Laura, ma, lontano da lei, non può fare a meno di cercare nell'aspetto delle donne che gli càpita di vedere lo stimolo visivo che gli permetta di ricreare dentro di sé l'immagine di lei (e solo nei limiti di questa attività rammemorante, nella forza interiore di questo fantasma o "idea" di Laura si può parlare, appunto, di "essenza platonica")».(A Fenzi si rimanda anche per la bibliografia ragionata sul sonetto). Non tanto, allora, perché le donne costituiscano un'approssimazione a Laura, piuttosto uno stimolo per l'astrazione della vera idea di Laura. Al limite, paradossalmente è meglio che Laura non ci sia, in presenza, davanti ai suoi occhi, perché è solo nella lontananza —e nella vicinanza fisica, viso a viso, delle altre donne, ma nell'impossibilità pratica di un confronto sulla scena reale - che è possibile il processo di astrazione verso la «forma vera». Quanto al ruolo della memoria, ne discuteremo più avanti. Maria Cecilia BerTolani, in Petrarca e la visione dell'eterno, Bologna: il Mulino, 2005, p. 176177, attribuisce a Fenzi l'ipotesi che «in altrui» valga «non tanto come "nelle altre donne", ma come "nel ritratto di Simone" ", con esplicito, diretto riferimento intertestuale agli altri due sonetti. Oltre che più sicure prove cronologiche, ciò comporterebbe una revisione ermeneutica. In realtà Fenzi: «Possiamo solo constatare che Petrarca, così come fa Dante [nella parte finale della Vita Nuova] si astiene per opportuno senso di discrezione dall'accostare il ritratto di Laura alla Veronica, cioè l'immagine doppiamente umana, perché dipinta da Simone Martini e raffigurante Laura, con l'immagine della Veronica doppiamente divina, perché raffigurante Cristo e da Cristo direttamente formata» (p. 33-34). E più avanti dissolvendo a monte ogni equivoco: "Al punto che in quel "in altrui" che pur giustamente va tradotto con: "in altre donne", mi piace di supporre, forzando il testo, proprio un'allusione al ritratto dipinto da Simone Martini, capace di alimentare all'infinito il desiderio senza mai trasformarsi in forma vera» (p. 38). Che «in altri» valga poi, addirittura, "nella Veronica stessa» (Maria Cecilia BERTOLANI, op. cit., p. 177) provoca un cortocircuito, forse fascinoso per le inchieste che accende, ma testualmente impraticabile. Infine: il collegamento, legittimo e persino necessario, di 16 alla questione del ritratto non necessita di forzare «in altrui». Ciò che cercheremo di dimostrare. 
cessori, ovvero il tema della pluralità degli amori, pur nella fedeltà dell'unico amore vero, per citare il Contini della letteratura delle origini, «" affermazione dell'unità dell'amore anche in presenza di passioni distinte, della radicale fedeltà metafisica nonostante l'apparente leggerezza», ${ }^{11}$ nella conferma dell'interpretazione del passo si misura già il salto: in Petrarca la pluralità delle donne si fa pluralità di immagini (di donne) che punge e rinvia all'unicità della visione di un archetipo ideale. Nelle mani di una tale «filosofo pratico», la storia dell'amore (e degli amori) si trasforma subito, di colpo, in storia di un procedimento mentale puro, del suo sforzo e anelito. Come si distingue dal Pellegrino (che non sceglie l'archetipo, archetipo e via per raggiungerlo gli sono già dati), così Petrarca si distingue da Cino e compagni: la fedeltà è un tributo alle proprie facoltà estetiche, non morali.

Se il sonetto non cessa di stupire per la pluralità di significati insiti nel parallelismo asimmetrico (asimmetrico a tutti i piani: metrico, sintattico, semantico) delle relazioni vecchierello/poeta, Veronica/altre donne, Cristo/Laura, non meno sorprendente e miracoloso risulta il rapporto tra l'umilissima epica religiosoodeporica della quête del romeo (al singolare, lui solo; perché? Lo vedremo documenti alla mano) e la condensata — in fondo aristocratica e solipsistica-, ma non meno dolorosa e laboriosa ricerca della forma vera: le sineddochi fisiche, l' «antiquo fianco», etc., si rovesciano in metafore intellettuali, «lasso», "quanto è possibile», ovvero in labor mentis. In questa specola si staglia il rapporto tra l'icona e il suo statuto di fruizione tipicamente medievale, da un parte, e il raggiungimento della forma vera attraverso il processo intellettuale, dall'altra. Detto ancor più schematicamente, ci troviamo di fronte alla ostensione, — non meno netta ed efficace se sceglie di presentarsi nella forma narrativa di un'esperienza che ne doppia un'altra, anch'essa narrata con scialo di particolari e senza il "come» incipitario del primo termine di paragone, piuttosto che in disposizione teoretica-, di un'opposizione; opposizione che non esclude un margine di contiguità, vedremo, tra due modi epocali di concepire l'immagine.

\section{Statuto delle Veroniche come immagini acheropite.}

Nel suo lungo excursus sulla natura delle immagini Jean-Jacques Wunenburger si sofferma sulla questione della mimesis, non senza citare la leggenda di Apelle, il ritrattista di Alessandro, che con pochi tocchi della mano sulla materia fece apparire la forma stessa di Afrodite, come un passaggio obbligato dell'origine mitica dell'immagine e della spiegazione dell'enigma dell'immagine stessa. Poi passa a distinguere le due logiche fondamentali antitetiche della mimesis, "una di riproduzione esterna, legata alla costruzione di opere sulla base di progetti (demiurgos), l'altra di riproduzione interna, di generazione o procreazione di un essere vivente per via naturale (phytourgos)». ${ }^{12}$ La prima appar-

11. Gianfranco Contini, Letteratura italiana delle origini, Firenze: Sansoni, 1993, p. 585.

12. Jean-Jacques Wunenburger, Filosofia delle immagini, Torino: Einaudi, 1999, p. 142 [Philosophie des images, Paris, Presses Universitaires de France, 1997]. 
tiene di diritto all'antichità greca e contribuisce alla formazione di una metafisica dell'immagine, particolarmente in Platone; la seconda viene rinnovata e approfondita in modo inaudito dalla teologia cristiana che la coniuga indissolubilmente alla fede e all'idea di un Dio incarnato: "Queste due logiche regoleranno - conclude lo studioso-, sia concorrenzialmente, sia sincreticamente, l'insieme delle variazioni concettuali e simboliche durante i secoli».

E ancora, a proposito della demiurgia delle immagini:

La concezione dell'immagine come riproduzione fedele di un modello deriva dal primato, fra tutte le attività umane, della fabbricazione di artefatti che riproducono doppi, sia di un'idea (realizzazione materiale del progetto di un oggetto), sia di una realtà fisica (ritratto dipinto di una persona). Ed è una concezione che, per analogia, fa da griglia di lettura anche per tutte le interpretazioni di genesi cosmica promossa dall'intervento di esseri divini o demiurgici (cosmogonie e teogonie). In entrambi i casi, infatti, la creazione appare come un processo attraverso il quale un progetto preliminare viene esteriorizzato e trasposto nello spazio-tempo di un'opera. Che emani da una divinità o da un demiurgo umano, la creazione consiste comunque nel fabbricare l'immagine di un modello e nel lasciarvi l'impronta e il marchio del suo autore.

La filosofia platonica fornirà una sintesi di questa concezione. «Nell'economia cristiana [invece, prosegue Wunenburger ${ }^{13}$ ] Dio, creatore increato del mondo, s'incarna egli stesso nel Figlio e genera una nuova immagine vivente di sé». Cristo è l'immagine visibile del Dio invisibile che in lui si è incarnato. "La teologia cristiana ha quindi optato per una superiorità del concetto di filiazione su quello di imitazione, al punto che per Sant'Agostino la vera immagine, ossia la più profonda somiglianza, si confonde con l'immagine dell'incarnazione cristica: "Ogni immagine — dice Sant'Agostino_ è simile a ciò di cui è immagine; tuttavia, tutto ciò che è simile a qualcuno non ne è anche l'immagine; per esempio, in uno specchio o in un dipinto le immagini in quanto tali saranno pure simili, e però se qualcuno non è nato da un altro, nessuno di loro può essere designato come immagine dell'altro. Infatti c'è immagine solo quando c'è discendenza 'espressiva' da qualcuno"». ${ }^{14}$

La preponderanza di fonti di parte vittoriosa, cioè iconofila, deprime l'importanza che ebbero anche presso molti scrittori cristiani fino al VI secolo e oltre le tesi iconoclaste, culminate nel precedente Concilio di Hieria (754). Lì, a Hieria, si teorizzò l'impossibilità teologica di raffigurare Cristo senza tradire il dogma calcedoniano della perfetta unione in una sola ipostasi della natura umana e di quella divina: la vera immagine deve essere consustanziale al modello. L'unica vera icona di Cristo è l'Eucarestia. ${ }^{15} \mathrm{E}$ «non ci son santi».

\section{Ivi, p. 151}

14. Ivi, p. 154. Per questa citazione da Agostino Wunenburger rinvia a Robert JAVELET, Image et rassemblance au XII.e siècle de Saint Anselme à Alain de Lille, Strasbourg: Universitè de Strasburg, 1967, p. 56. V. anche Maurizio Bettini, Tra Plinio e Sant'Agostino: Francesco Petrarca sulle arti liberali, cit., p. 234.

15. Ivi, p. 175. 
Al Concilio niceno secondo si rispose (e i toni accesi, come si è letto, dicono della consapevolezza di un destino storico) agli iconoclasti che «l'immagine di Cristo è in rapporto con la persona secondo il nome, e non secondo la sostanza: l'icona non è il Cristo, ma lo rappresenta sulla base della verità storica dell'Incarnazione». ${ }^{16}$ Il più strenuo difensore delle immagini, Giovanni Damasceno (675-749) le difende con l'argomento della sola Incarnazione: "E' impossibile rappresentare in immagini ciò che è incorporeo, privo di forma, invisibile, e neppure circoscritto... E' da Dio fatto carne, veduto sulla terra in carne e ossa e vissuto tra gli uomini con la sua ineffabile bontà, è dal Dio che ha assunto la natura, lo spessore, la forma e il colore della carne, è da lui che noi ricaviamo un'immagine». ${ }^{17}$ Spiega definitivamente Jean-Luc Marion:

L'incarnazione che esprime la persona di Cristo e la natura divina non prolunga la presenza di tale natura se non nell'Eucarestia, in cui non interviene alcun volto; specularmente, essa legittima l'immagine-icona, volto perpetuo di Cristo, senza che vi intervenga sacralmente la natura divina. ${ }^{18}$

L'icona fonda la giustificazione del suo statuto teologico sull'hypostasis: Dio vi è venerato (non adorato) per presenza ipostatica, non per presenza della natura divina come nell'Eucarestia.

L'immagine acheropita, invece, si promuove proprio come una nuova cristofania e come tale miracolosa e salvifica. Per tornare al vecchierel: non si dice, nel sonetto, perché si dà per scontato, che scopo del suo itinerario verso la contemplazione dell'icona acheropita, sia la Salvezza. Mentre per Petrarca... Se si occupasse di tali questioni, il vecchierel sottoscriverebbe le considerazioni di von Schönborn: «L'icona acheiropoietica costituisce per così dire il luogotenente, il tenente-luogo dell'Uomo definitivo, di quest'uomo a venire, ma attesta al tempo stesso, con la propria presenza e il proprio realismo, che quest' Uomo definitivo è già venuto, si è già reso visibile in un volto umano». ${ }^{19}$

E Petrarca...? La soggettività moderna ha altre gatte da pelare.

\section{Icone su e giù per il Mediterraneo.}

Due icone concorrenti, nei due sensi della parola, si aggirano nel Mediterraneo e finiscono per sovrapporsi nel comune statuto che le lega: la Veronica in San Pietro e il Mandylion di Edessa. Prevarrà la prima. La Vera Icon, come suona con splendido slogan da autentici «creativi» la pseudorietimologizzazione medievale (probabilmente Veronica deriva dal nome greco Berenice, Bernike o Phe-

16. Ivi, p. 181.

17. Giovanni Damasceno, Discorso apologetico, cit. da Jean-Jacques WuneBURGER, op. cit., p. 222.

18. Jean-Luc MARION, Le prototype de l'image, in François Boespflug e Vladimir Lossky (a cura di), Nicée II, Paris: Cerf, 1987, p. 462 (cit. da WunENBURGER, op. cit., p. 216).

19. Christian VON SCHÖNBORN, Les icones qui ne sont pas faites de main d'homme, "Image et signification", Paris: La Documentation Française, 1983, p. 211 (cit. da Wunenburger, op. cit., p. 219). 
renìke, "portatrice di vittoria»). ${ }^{20} \mathrm{Ma}$ è la prima quella che racchiude una più antica storia esemplare da cui la seconda poi prende slancio. ${ }^{21}$ Tutte le innumerevoli riproduzioni e "parafrasi» del Mandylion si rifanno a un originale che non era propriamente una icona ma un panno. ${ }^{22}$

Dentro la plurima diegesi ${ }^{23}$ che l'accompagna e spesso viene rinarrata figurativamente mediante formelle o altro, si potrebbe scegliere il seguente racconto come il più ricco di implicazioni apologetiche e didascaliche.

Malato di lebbra e prostrato nel proprio letto il re Abgar di Edessa, non potendo raggiungere con le sue gambe Gesù, invia al Salvatore il messaggero Anania. Anania è anche un pittore e arrivato davanti a Gesù tenta di rappresentarne il volto con i pennelli. Invano. Non può dipingerlo e, nel suo tentativo di riproduzione mimetica, fallisce. Impietosito, Gesù si fa portare dell'acqua da un servo, lava il proprio viso e mentre lo asciuga lascia per miracolo l'immagine del volto sul telo. Questa immagine-reliquia giunge al re Abgar e lo guarisce. Il re si converte al cristianesimo. Accanto a questo episodio fondamentale ce ne sono altri che raccontano la genesi delle «reliquie per contatto". Nessuna leggenda è più sinteticamente apologetica di questa in fatto di teoresi sull'immagine sacra. L'icona traccia intorno a sé una linea netta di confine stabilita dal limite del pittore Anania, reso impotente di fronte al divino, inutili i suoi pennelli. L'icona è sacra per contatto con il divino, "è» il divino, volto divino essa medesima. Il carattere acheiropoietico sospende ogni eventuale accusa di iconodulia. Motivo comune a molti racconti sulle icone, il fallimento del pittore mette ulteriormente al riparo. ${ }^{24} \mathrm{D}$ 'altra parte l'assenza di mano umana garantisce all'opera la qualità della reliquia e dunque la possibilità di riprodursi all'infinito per contatto (anche tra materiali diversi: dal panno alla ceramica): ${ }^{25}$ così si ribadiva l'indipendenza dalla materia.

20. Mi valgo dei contributi inclusi in Giovanni MorelLo e Gerhard WOLF (a cura di), Il volto di Cristo, a cura di, (Catalogo della mostra "Il volto di Cristo», Roma, Palazzo delle esposizioni 9 dic. 2000-16 aprile 2001), Milano: Electa, 2000; e di quelli contenuti in Mandylion. Intorno al Sacro Volto, da Bisanzio a Genova, a cura di Gerhard Wolf, Colette Dufour Bozzo, Anna Rosa Calderoni Masetti, (Catalogo della mostra «Mandylion. Intorno al "Sacro Volto" da Bisanzio a Genova», Genova 18-aprile-18 luglio 2004), Milano: Skira, 2004; e di Hans BELTING, Il culto delle immagini. Storia dell'icona dall'età imperiale al tardo Medioevo, Roma: Carocci, 2001. Altri studi sono cit. da Rosanna BeTTARINI, ediz. cit., p. 74. E da Silvia CHESSA, Il profumo del sacro nel Canzoniere di Petrarca, Firenze: Società Editrice Fiorentina, 2005, p. 45.

21. Altra, non meno complessa, è la vicenda materiale: «Il mandylion fu trafugato dopo il 1204 o fu distrutto a Parigi? Oggi si trova a Roma o a Genova? La Veronica è sparita a Roma all'inizio del Cinquecento, o fu venduta all'incanto dai soldati di Carlo V dopo il sacco del 1527?»: Gerhard WOLF, Dal volto all'immagine, dall'immagine al volto, in AA.VV., Il volto di Cristo, cit., p. 20.

22. "L'icona non può essere definita esclusivamente come pittura su tavola. Essa non rappresenta una tecnica pittorica, ma un concetto di immagine destinata alla venerazione»: Hans BELTING. Il culto dell'immagine, cit., p. 47.

23. Herbert L. Kessler, Il mandylion, in AA.VV., Il volto di Cristo, cit., p. 67.

24. Michele BACCI, La fisionomia di Cristo nelle testimonianze letterarie del Medioevo, in AA.VV., Il volto di Cristo, cit., p. 33.

25. Si vedano gli smalti della cornice eseguita nel XIV secolo per il Mandylion di Genova (Chiesa di San Bartolomeo degli Armeni), in cui viene narrata la serie dei miracoli operati dal 


\section{La Veronica. Storia di un successo teomediatico}

Non dissimile, almeno nel nucleo funzionale, la storia della Veronica. Nome che designa sia il sudario di San Pietro sia la proprietaria originaria, la (apocrifa) Santa Veronica ${ }^{26}$ la cui più antica fonte risale al $\mathrm{X}$ secolo. Il primo autore a testimoniare la fortunatissima etimologia di Veronica come «vera icona, id est, imago vera» è Gervasio di Tilbury un pellegrino inglese diretto a Roma nel 1200 ca., nei suoi Otia Imperialia, dove racconta che Veronica offrì il proprio velo («peplum») a Cristo, il quale lo calcò sul viso («impressit») lasciandovi impressa la propria immagine («imago expressa»). ${ }^{27}$ Ma le scadenze storiche più importanti, anche per noi accaniti archeologi di $R v f 16$, sono riassumibili in tre "giornate»:

1) la fondazione del culto della Veronica da parte di papa Innocenzo III (11981216), che istituì una processione annuale che voleva essere una commemorazione liturgica delle Nozze di Cana durante la festività dell'Epifania. I pellegrini si avviavano all'incontro con la Veronica come a un rito nuziale. Il vecchierel lascia la famigliola sbigottita per un altro sposalizio trascendente. E Petrarca...

2) la centralità della Veronica nell'anno del Giubileo (1300), quando la facies di Cristo è la méta ultima dei romei (con anticipazione in Vita Nuova, XL: «Avvenne, in quel tempo che molta gente va per vedere quella ymagine benedecta la quale Gesucristo lasciò a.nnoi per exemplo della Sua bellissima figura, la quale vede la mia donna gloriosamente, che alquanti peregrini passavano...») come culmine del processo parallelo di sviluppo del culto della Veronica insieme con la fissazione del dogma della Transustanziazione (1215) e la introduzione della festa del Corpus Domini (1264).

a) 1337: con la Bolla di Benedetto XI, Benedictus Deus, il concetto di giudizio individuale viene elevato a dottrina della Chiesa. Se il Giudizio coinvolge il destino del singolo, l'incontro ultimo alla fine della vita e alla fine dei secoli con il volto di Dio, sarà un evento squisitamente individuale. Muta quindi il significato dell'esperienza personale davanti all'immagine: il «vecchierel» viaggia non in comitiva, con un inclusive tour specializzato, ma solo. E solo arriva davanti alla Veronica, solo pensa al Volto "ch'ancor lassù nel ciel vedere spera». Solissimo poi quel poeta che tenti di doppiarne quell'esperienza altrimenti, con mezzi da laico pellegrino intellettuale «fai da te».

sacro volto, per esaltare il potere della reliquia: Mandylion. Intorno al Sacro Volto, da Bisanzio a Genova, cit.

26. Probabilmente somma di più personaggi femminili, inclusa l'Emorroissa di cui parlano i Vangeli apocrifi e $M t$ IX, 20-22; $M c$ V, 25-34; Lc VIII, 43-48. Cfr. Gerhard WOLF, "Or fu si fatta la sembianza vostra?" in AA.VV., Il volto di Cristo, cit., p. 103 sgg. A differenza della tradizione veterotestamentaria e ebraica, prevalentemente "orale», il cristianesimo iconofilo sviluppa pro domo sua il punto centrale del ritratto.

27. Gerhard WOLF, "Or fu si fatta la sembianza vostra?», in AA.VV., Il volto di Cristo, cit., p. 105. 
E il 1337, neanche a ricordarlo, — neanche a farlo apposta per raccordare le contingenze storiche-, è l'anno attorno al quale la filologia fa confluire $R v f$ $16,77,78$.

La soggettività, proposta in termini inauditi in $R v f$, e comunemente accreditata con l'attributo di «moderna», passa per tale processo graduale e può alludere, pure, alle progressive modificazioni istituzionali religiose del medioevo. Farne leva.

Nel momento in cui propone, —-mettendo in scena diegeticamente la Veronica e il suo statuto_-, la legittimità dell'estrazione dell'idea, dell'immagine vera, dalla molteplicità dell'esistente, e la coltivazione della medesima, sulla base di una prassi personale forzata, quasi un "non poterne fare a meno» insomma una specie di coonestazione della raffigurazione dell'idea pura nell'intelletto-, Petrarca pone le basi, almeno, per un'autonomia di quella stessa raffigurazione virtuale. Pone le basi per il ritratto laico moderno:

Il ruolo catalizzatore della vera icona nella nascita del ritratto moderno corrisponde al suo ruolo nella devozione individuale, ossia nell'incontro del singolo con la vera immagine di Cristo. ${ }^{28}$

Ovviamente la nascita del ritratto moderno non è segnata da uno strappo o dal semplice voltare pagina alla fine del capitolo medievale della storia dell'arte, presuppone invece una parabola che si spicca dal ruolo e dall'incontro del singolo con la Vera Immagine. Nel caso nostro: indagine e discorso su volto e ritratto di Laura non possono darsi se non attraverso la connessione con il lungo discorso e dibattito storico teologico sulla venerazione del volto divino. Ne va della legittimità dell'oggetto.

\section{Due romei e il sacro come profumo}

Prima di affrontare 77 e 78, s'impone la questione: se e in che misura i tre sonetti si assestino su una linea avanzata rispetto ai millanta riferimenti alle arti liberali sparsi altrove. $\mathrm{O}$, che è lo stesso quesito rivoltato e allargato a ventaglio: se e in che misura la questione del ritratto riproponga la caratterizzazione dello status culturale «laico» di Petrarca.

Ma forse c'è un errore originario: non ci eravamo accorti che il Petrarca di $R v f 16$ era in realtà un asceta religioso. E non un'intellettuale lanciato verso orizzonti inesperiti della cultura più avanzata dell' homo sapiens sapiens.

Avanza, infatti, chi il "profumo del sacro» lo avverte con narici sensibilissime, sparso come incenso denso in tutto il Canzoniere. ${ }^{29}$ Il che riapre di colpo la questione della laicità, anche se — sperabilmente - su un fronte nuovo e più avanzato.

28. Gerhard WOLF, «Or fu sì fatta la sembianza vostra?», cit., p. 112.

29. Silvia CHESSA, Il profumo del sacro nel Canzoniere di Petrarca, Firenze: Società Editrice Fiorentina, 2005. 
Nel corposo volume di Silvia Chessa, densissima e acribica ricognizione delle fonti patristiche, un intero capitolo è dedicato a $R v f 16 .{ }^{30}$ Ovvero pure XVI emanerebbe profumo di sacro, eccome, da tutti i pori del testo.

Non ogni sentenza, nel complesso e ricco discorso, è perspicua: «Facile ed erronea l'identificazione di forma vera e veronica che calamita sul piano interpretativo quella di Cristo-forma vera e donna amata, ulteriore complicazione del dotto garbuglio di leggende sulla donna che il tempo ha battezzato Veronica e la Chiesa latina ha santificato». ${ }^{31}$ "Identificazione»: da parte di chi e, soprattutto, in che termini?

Vediamo gli assunti. 16 si iscriverebbe nello «spazio teologico» di una «fabula (an historia?) sacra», «La rielaborazione cristologica delle vestigia Laure testimonia visibilmente l'approdo sicuro della doppia ricerca, poiché il cielo è la sede del doppio sole, la donna e Dio: in "Quel sol che mi mostrava il camin destro", con quel che segue; "forma vera è l'oggetto filosofico e mistico di un'esperienza ripetutamente e ontologicamente fallimentare, non la forma perfetta o un generico eterno femminile, ma l'eterno di Laura. La sua anima»; «e solo il cantiere aperto dall'esegesi agostiniana permette il recupero della pur visibilissima sinopia, la sopracitata Fam. II, 9, da cui riverberano serialmente nel sonetto 16 la gradualità tra materiale e spirituale, la scalarità fra terreno $\mathrm{e}$ celeste, il trinitarismo concettuale applicati a Roma», etc., etc. ${ }^{32}$

Iniziamo dal fondo. La Fam. II, 9 è un sunto delle «dolcezze» proprie del cristiano: «quam dulce tamen est cristiano animo urbem cernere celi instar in terris», e poi «videre verendam populis Salvatoris imaginem et in saxo durissimo eternum gentibus adoranda vestigia», con tanto di aggiunte della citazione di Isaia, «Et venient ad te curvi filii eorum qui humiliaverunt te». Si può sostentere che il semplice accenno alla «verenda imago Salvatoris» sia panoplia di 16 ? 16 per intero, nella sua fulminea complessità? O questa, come altre, è l'occasione propizia per toccare con mano come Petrarca si valga di fonti culturali quale materiale di nobile edilizia per costruire un altro edificio e andar oltre la loro originaria struttura culturale, oltrepassarle, in qualche modo concellarle?

Anche sul rimanente del discorso e delle figure petrarchiane Chessa intesse una fitta trama di riferimenti biblici e patristici in una pluralità di rivoli digressivi e di ardite giunture, ${ }^{33}$ ottenendo una serie di tessere che, alla fine, riconducono l'esegesi del testo a storia catalogico-citazionale, pronte per essere ricomposte a configurare una storia mistica (inclusa, s’è visto, la forma vera) e sacrale (fabula sacra).

Sul piano macrotestuale, per di più, si tende a spiegare 16, in linea generale, con i testi più tardi (Rvf306, "Quel sol che mi mostrava...», etc.) allorché, «in

\section{Ivi, cap. II.}

31. Ivi, p. 45. L'ulteriore complicazione del «garbuglio» meriterebbe un tentativo, limpido, di sbrogliarlo.

32. Ivi, p. 46, 53, 57, 62-63.

33. "Il viator è l'autore, pellegrino, "uomo di pena" ancora in Ungaretti, mortale che strascica la propria "carcassa / usata dal fango / come una suola / o come un seme / di spinalba" (Pellegrinaggio)»: ivi, p. 70. 
coda al Canzoniere Laura opera di sua mano una riCreazione mirata alla comprensione, alla gratitudine e all'ossequio per l'atto gratuito, per un altro sacro vestigio, la prima impronta, quella divina che rende molto simili a Sé ( $G n$ I 26-27, V i, IX 6, Sap II 23, Sir XVII 1, Col III 10)», con citazioni di 360, 127 30; allorquando emergerebbe, insomma, la "cristica umanità di Laura». ${ }^{34}$ "In altrui» sarebbe allora riconducibile alla "vacua pulchritudo corporis», conforme al dettato salomonico in chiusa ai Proverbia, "fallax gratia et vana est pulchritudo» (XXXI 30), citato nel De ignorantia (II 13). Appartiene alla rubrica dei carnalia, sensibilia, visibilia, temporalia, mentre donna, cioè Domina, sarebbe il paradigma della distanza e dell'inattingibilità, il contraltare del Dominus oggetto della quête, scopo dell'itinerarium mentis e complemento naturale della visio dei, teste la prima terzina di Volo con l'ali de pensieri al cielo (Rvf362, 911). ${ }^{35}$ Dunque, sempre per via di incroci vettoriali di citazioni (e sempre con i Fragmenta a più alto ordinale), la quête del romeo e quella del poeta vengono a convergere in un unico traguardo religioso e mistico, coppia di figurine sacre sovrapposte fino alla coincidenza. ${ }^{36}$

Da tale dissolvenza incrociata, fondu dei ritratti dei due personaggi finalmente gemellati in un unico pellegrinaggio, si deduce la conclusione che attiene al maggiore, il Soggetto in causa: "Il possesso di Dio risponde a un eros che palpita e la tentazione al trascendente non è solo un'inquitudine psicologica e gnoseologica, è un'angoscia radicale che si placa solo in Dio: finché non si raggiunge l'oggetto della ricerca, il cuore è agostinianamente inquietus, e l'inquietudo è la sua infelicità. Così Petrarca confessa il dolore di un iterato e angoscioso vano sforzo per corporalia ad incorporalia nel ricordo parallelo della passione cristiana e della sua non meno dolorosa metafora erotica. Ma la similitudine non quadra al Tassoni, al Muratori, al De Sanctis: il rapporto fra i due termini della comparatio, falso nodo della questione, si trasforma in un laccio esegetico che intrappola illustri esponenti della nuova e ben armata militia Petrarce». Nessuno si sarebbe accorto, insomma, dell'anagoge. ${ }^{37} \mathrm{E}$ l'eros è bell'e asservito alla mistica.

Se così stanno le cose, l'ermeneutica di matrice mistica (ma che significa «ricordo [...] della sua non meno dolorosa metafora erotica»? Riduzionismo

\section{Ivi, p. 57.}

35. Ivi, p. 66.

36. Da una parte la vicenda del vecchierel è psicologizzata fino ad approssimarlo alla configurazione antropologica in questione nei Rvf: «la senilità fisica [...] è la veste del vetus homo, il peccatore paolino e agostiniano che tenta di emendare il "giovanile errore" $(\operatorname{Rvf} 1,3)$, anch'esso agostiniano». Dall'altra l'itinerario del Petrarca pare ricalcare passo passo le orme del romeo come fossero davvero compagni di un unico viaggio. Ivi, p. 68. Con cit. di $L c$ IX 61-62, «in cui l'evangelista offre il destro alla "stigmatizzazione" dello sguardo rivolto indietro al peccato, al mondo e agli affetti familiari» [Ivi, p. 71; ma dove è rimarcato esplicitamente, in XVI, che il romeo parta per purificare i suoi peccati?]. Fessus, inscius, occupatus è il peccatore Petrarca (De otio I, p. 570), lo straniero che desidera compiere l'ultimo tratto della propria vita mortale e riposare la propria stanchezza esistenziale a Roma (Fam. XV 87 e XXIII 9 1).

37. Ivi, p. 73. 
sbrigativo delle valenze anche culturali e letterarie dell'erotismo? Negazione della capacità di Petrarca di valersi dell'ossessione erotica per far progredire il discorso fino all'estremo storico possibile?), l'interpretazione non sarà poi tanto distante da quelle analoghe similari, che so, da quella «ascetistiche» di Barberi Squarotti, già confutata definitivamente da Fenzi, ${ }^{38}$ che individua anche in Petrarca, come nel romeo, lo sforzo di un itinerario ascetico ad Deum. ${ }^{39}$

$\mathrm{Al}$ di là dei possibili romanzi religiosi citazionali, più importante è la questione della resa finale di "forma vera». "Vera» equivale a «reale», quella vostra, di voi, donna lontana? O quella ultraterrena? E in che senso: «anima» o «idea»? Chessa sostiene, —s'è visto- l'equazione forma $=$ anima ${ }^{40} \mathrm{Ma}$ nel finale appena citato del suo capitolo ad hoc, pare alludere a un diverso rapporto tra i due termini, «falso nodo della questione», che avrebbe ingannato generazioni di esegeti. Dunque, diversa semantica per annullare tutto in una identificazione?

Eppure se la forma vera è res intelligibilis, come concludono in tandem Bettarini e Chessa su XVI, ${ }^{41}$ —res intelligibilis in opposizione a imago, l'intelligibile eterno si dà proprio come forma, species, idea dentro l'impresa «non nuova per Agostino di vestire il platonismo di cristianesimo»—, ${ }^{42}$ la res intelligibilis può ben vantare uno statuto riconducibile al paradigma squisitamente visivo. "Anima» sarà piuttosto eidon.

Del resto che la forma vera racchiuda in sintesi la virtuale esperienza ottica, sia pure di un occhio della mente (frustrata), o dell'anima (un bel giorno), si evince pressoché lapalissianamente dal parallelo con l'icona della Veronica, che è appunto un dipinto, agognato dalle masse dei pellegrini che intendono esperirlo innanzitutto con l'occhio, teste sempre Dante ("Or fu sì fatta la sembianza vostra?»). E poi: perché mai il Soggetto, dopo aver contemplato il romeo che contempla la Veronica, dovrebbe cercare l'invisibilium eterno, e non invece una res, un'idea potenzialmente visibile?

Nello slancio ansioso, nello sforzo, costante in $R v f$, di astrarre per via di pensiero raffigurante (e prendiamo pure CCCXIX, 9-14: «Ma la forma miglior, che vive anchora, / et vivrà sempre, su ne l'alto cielo, / di sue bellezze ognor più m'innamora; / et vo, sol in pensar, cangiando il pelo, / qual ella è oggi, e "n qual parte dimora, / qual a vedere ilo suo leggiadro velo" (corsivo mio), sta la novità. La novità sta, appunto, nello sforzo del pensiero puro immaginante: nella concentrazione del «disegnare con la mente».

38. Enrico FENZI, Note petrarchesche: Rvf 16, cit., p. 19.

39. Giorgio BARBeri SquarOtti, Il vecchio romeo: Petrarca, 16, "Critica letteraria», xxii, 1994, p. 43-52.

40. Ivi, 57 e n., 58-60.

41. Francesco Petrarca, Canzoniere Rerum vulgarium fragmenta, a cura di Rosanna BetTarini, cit.: «La forma vera, più che la "platonica essenza della donna" (Velli), è la res intelligibilis, l'anima che "sine ulla dissimilitudine" s'identifica con Dio" (p. 75, citando Chessa; e v. p. 70-71). E cfr. poi il commento a Rvf 129, 72, 126, 60.

42. O non sarà meglio invertire i termini e parlare di cristianesimo vestito di platonismo? Sul platonismo di p. non c'è spazio qui per riflettere; si rinvia, intanto, a Enrico FEnZI, Platone, Agostino, Petrarca, in Saggi petrarcheschi, cit., p. 519-551. 


\section{Perché novità c'è.}

La poesia — non solo 16 — non può ricondursi alla somma delle sue fonti (probabili, possibili, e magari —almeno quantitativamente- impossibili). Altrimenti l'intero Canzoniere apparirà solo come una sintesi, sia pure costituita di mirabili incastri e incastonature da un prodigioso cesellatore della cultrua universale, di sintagmi, sentenze, definizioni e precettistica dei santi maestri, già del resto reperibile nella trattatistica in prosa del medesimo autore. Al contrario, il genere stesso, la poetica del fragmentum, il «frammento poetico", insomma la lirica, e la formula metrico-retorica dei singoli componimenti — non esclusa la stilizzazione, come qui, quella del romeo, personaggio non psicologizzabile - con l' autorizzazione di un retaggio ormai secolare, garantisce la fondamentale libertà di andare oltre i sacri testi di riferimento, classici o cristiani. Di bruciarli nell'esatto momento in cui li si adibisce.

Prova prima e ultima si può reperire puntualmente nei nostri 16 (e nei gemellati 77,78 ) in cui è ben difficile, stando alla lettera del dettato, cogliere la pur minima concessione al rilievo della questione morale (la peccaminosità del desiderio che insegue il volto e la pulchritudo di Laura in raffigurazione mentale o in rappresentazione figurale concreta), laddove i trattatelli di un Petrarca che non è uguale a se stesso, assorbono e rinviano in pieno le discussioni etiche pregresse. Perciò Canzoniere, sì, per riconoscimento universale di paradigma lirico. Rvf, certo, per precisa e sottile avvertenza del Soggetto medesimo che si riconosce pure nella struttura dell'oggetto poetico e in essa giustifica la forza della sua recherche. Non «Novus liber confessionum», cioè trattato autobiografico-morale ideologico, parabola progressiva di romeo unidimensionale. ${ }^{43}$

\section{Rvf 77}

Nella parafrasi che offriamo in nota ${ }^{44}$ si sottinende che la congiunzione "Ma", avversativa e argomentativa, racchiude in sintesi: ma la questione non è quel-

43. La definizione è di CHESSA, op. cit., p. 58; appoggiata a De otio II, p. 802, che è un trattatello, per l'appunto.

44. Anche se Policleto e gli altri (artisti) che ebbero rinomanza in quell'arte (figurativa, della rappresentazione del corpo umano), gareggiando tra loro, fissassero tutti assieme lo sguardo attento per mille anni di seguito (sul volto di Laura, o meglio: sull'idea intelleggibile della bellezza del suo volto), non vedrebbero che una parte minore della bellezza che ha preso possesso del (conquistato e vinto il) mio cuore. Ma certamente il mio Simone (mio amico; e artista da me eletto) fu in paradiso da dove questa nobile donna proviene direttamente. Lassù (in paradiso) (Simone) la vide e ne fece il ritratto su pergamena per poter testimoniare qua giù (in terra) della bellezza del suo viso. L'esecuzione dell'opera figurativa (il ritratto) fu davvero di quelle che si possono concepire in cielo, non (qui in terra) tra noi (mortali), dove il corpo fa schermo all'anima. Fu un atto di cortesia (di generosità) ritrarla (e donarmi l'opera); né (Simone) avrebbe potuto eseguire (il ritratto) dopo che fu ridisceso giù (in terra) a percepire (la realtà concreta) con il corpo sensibile e dopo che il suo sguardo partecipò (di nuovo) delle cose mortali (fisiche, materiche). 
la del raffronto con Policleto, né quella della bellezza in sé o della sola bellezza di Laura, piuttosto la questione dell' attingimento mediante una procedura straordinaria che ora vi spiego; «ma certo» = ma occorre dire, per spiegare tale superiorità, che l'esperienza, il procedimento eccezionale esperito da Simone fu...

Sul piano generale, emergono:

a) il richiamo al confronto con la classicità (Policleto, mitico scultore, lo scultore classico per antonomasia nelle classifiche medievali) non si svolge sul piano delle procedure tecniche; ciò che li divide sono due diversi approcci visivo-mentali, differenti per livello qualitativo: Policleto potrebbe osservare per mille anni il volto terreno di Laura... Simone ha osservato ben altro. E altrove.

b) La sottesa superiorità del moderno sull'antico. Simone, che «fa fede quaggiù del suo bel viso» vince Policleto e gli altri, dunque sarà degno di maggiore o almeno ugual fama.

Sul piano dell'esecuzione:

a) L'oggetto non è l'intero corpo, come più spesso nella scultura, ma è il volto («bel viso») secondo una tipica moderna identificazione del volto con la persona che certo affonda le radici anche nella cultura stilnovistica e dintorni, ma per superarla. Insomma, il volto come sintesi della persona (tipicamente occidentale: altre culture —culture «altre»— rivendicano che «il volto sta in ogni parte del corpo»). Ovviamente il processo ingloba la passata esperienza epocale per cui il volto di Cristo-Dio rappresenta pienamente l'incontro dell'umano con il divino; processo che, una volta laicizzato, può garantire al viso dell'uomo la conservazione di alcuni attributi di nobiltà e di qualità assoluti sopra ogni altra figura naturale.

b) Il processo intellettuale presuppone un itinerario dalla terra al cielo e dal cielo alla terra ${ }^{45}$ di cui vedremo fra poco i corollari.

c) Anche se esiste l'enorme precedente di Purgatorio XII, 65-67 «Qual di pennel fu maestro o di stile / che ritraesse l'ombre e' tratti ch'ivi / mirar farieno uno ingegno sottile?», dove "ritrarre» è impiegato proprio in senso tecnico (o per pennello o per stilo), Petrarca riadibisce puntualmente il termine nel senso tecnico, e per di più riferito non più a masse e lineamenti («ombre» e "tratti» cioè appunto «masse» e «lineamenti generali») collettivi, come Dante, ma a un viso, un volto, ovvero a ciò che solo nel Cinquecento si designerà con il deverbale «ritratto». «In carte», ovvero su pergamena (miniata) (Purg XI, 82: «Frate, più ridon le carte / che pennelleggia Franco Bolognese»). "Carte» vale anche, si sa, per «scrittura su carta» (pergamena), ovvero per antonomasia interna, «i versi» («Però mi dice il cor ch'io in carte scriva / cosa, onde 'l vostro nome in pregio saglia", 104, 5-6). Paral- 
lelamente «stile» (78): «Quando giunse a Simon l'alto concetto / ch' a mio nome gli pose in man lo stile», "verghetta di piombo e stagno o d'argento per disegnare», ma pure «né col mio stile il suo bel viso incarno» $(308,8)$, insomma il "vario stile» di $R v f 1$, stilistica come realizzazione tematica e morale delle Rime, scrittura in versi con i suoi timbri e toni. Infine: somma di tecnicismi ambivalenti, e per la scrittura e per la pittura. Coonestazione letteraria intralinguistica della terminologia tecnica. A conferma che anche nella strumentazione può sussistere equivalenza tra arte liberale (pittura) e poesia. Nel regno di Amore, s’intende. Nonché di amicizia («il mio Simon»). ${ }^{46}$

d) «Cortesia fé» pare la variazione figurale di «Simon Fecit». Qui il soggetto per metonimia allegorizzata è l' atto di «liberalità», atto di nobile generosità che investe anche il manufatto.

Come si realizzò la metonimia? Eseguendo e regalando il ritratto, ma anche percorrendo l'itinerario dal terreno al superno, comunque un atto eminentemente libero, non necessario com'è —invece- il miracolo teologico che giustifica l'icona. Simone non trascina dolorosamente il suo fianco fino a Roma, balza agilmente in Paradiso perché ha portato fino a compimento, dentro la mimesi artistica, quel percorso teorico che il poeta era riuscito in un lampo, in via propedeutica e precorritrice, a sintetizzare in terra e nella mente quando aveva desunto dalle varie forme terrene donnesche la vera forma dell'unica sua donna. Se la provenienza paradisiaca di Laura («onde questa gentil donna si parte») può essere considerato richiamo stilnovistico strumentale al discorso sul procedimento artistico (e la nobiltà della donna gentile si riverbera allora sul processo di intellettualizzazione delle idee come enti visivi), l'ascesa del pittore in Paradiso non avrà l'unico scopo di suffragare la sua superiorità in base al privilegio cristiano sul pagano (Policleto), —una vittoria che risulterebbe pur sempre acquisita per rendita passiva-, ma ancor più laicamente alluderà al mondo medesimo delle idee, cioè con puntualità alla spiegazione precisamente neoplatonica, di cui già a suo luogo.

Petrarca discute meno (o quasi per nulla) degli aspetti del ritratto che del suo statuto dentro la riflessione implicita sulla verità dell'immagine, cioè dell'eidon.

46. Altrove p. usa altri tecnicismi. Ma significativamente in 77,78 solo quelli bivalenti, pronubi per pittura e poesia. Per spiegare in che consista la somiglianza fra padre e figlio, nella Fam. XXIII, 19.11 Petrarca parla di «un certo adombramento, quello che i nostri pittori chiamano "aria" [aer] ed è visibile soprattutto negli occhi e nel viso"; un termine effettivamente in uso nella pittura (Maurizio BETTINI, op. cit., p. 235-236). "[aer] è voce di lessico tecnico, in due accezioni che possono fondersi: il quid individuale d'uno stile e l'espressione di volti dipinti o scolpiti [...]. La seconda accezione — l'"aria delle teste" o del "viso"è endemica in Vasari: Petrarca la riconduce al gergo dei pittori trecenteschi: ed è notevole, credo, che da lì filtri nel lessico distillatissimo del canzoniere - "quell'aria dolce del bel viso adorno" (122,13) — e quindi nella nostra lingua».(Maria Monica DONATO, op. cit., p. 444. Più volte utilizzò «penicillus» "pennello». 
Lintellettuale-poeta «inesistente come critico d'arte» (secondo Contini; di contro al Dante fieramente tassonomico dei grandi nomi di artisti, che inaugura la critica d'arte, secondo Longhi) ${ }^{47}$ fuori del Canzoniere avalla la possibilità e legittimità di una storia dell'arte, ${ }^{48}$ rifiutando però ancora una volta la pura teoresi compatta, e dentro il Canzoniere si propone come teorico affabulante, in versi di omaggio, del processo di mimesis: premessa storica e fenomenologica (16), percorso e risultati (77), effetti (78) dell' iter mimetico. Porta così in avanti il processo di identificazione tra «ideazione» ed «esecuzione» che sancisce il passaggio tra medioevo (nell'icona la concezione ideativa era appannaggio dei santi padri, la mera realizzazione materiale degli artefici) e età moderna. Né accenna a questioni di abilità tecnica, di mimesi «realistica», di «verosimiglianza». Punta a esaltare la performance dell'artista che pertiene a una ascesi intellettuale che ha del miracoloso.

Dà risposta alla domanda di 159, 1-3: «In qual parte del ciel, in quale ydea / era l'exempio, onde Natura tolse / quel bel viso leggiadro, in ch'ella volse / mostrar qua giù quanto lassù potea?»

A rigore Simone (l'Arte) segue passo passo la Natura, cioè l'ordinamento cosmologico. E attinge, sì, finalmente, la parte più nobile del Cielo in cui sta un simile modello. D'altra parte: assistiamo passo passo al progressivo scambio tra «produttore» e «fruitore».

\section{Dal produttore al consumatore}

Anche il rapporto visibilità/invisibilità che era alla radice delle discussioni teologiche filoclastiche o iconoclastiche sulla rappresentabilità del volto di Dio, per cui il volto umano di Cristo, per i filoclasti, rinviava all'invisibilità di Dio nel momento stesso che ne realizzava la visibilità grazie alla doppia natura cristologica, qui viene declinato - ancora neoplatonicamente— sul piano delle forme impure terrene («membra», «velo», «caldo et gielo», «del mortal sentiron») e forme pure iperuraniche (il paradiso delle idee, comprese quella della beltà del volto di Laura). Per cui se nella storia profilata dal Canzoniere la Laura paradisiaca di Simone entra in gioco puntualmente proprio dopo la «svolta spiritualizzante» ${ }^{49}$ delle canzoni $70-73$ (che tre di loro siano le cantilene oculorum farà al caso nostro, ossia rimarcherà l'approfondimento del significato dello sguardo applicato infine alla sua arte specifica come al suo traguardo e svelamento finalmente attuato), la «spiritualizzazione» di Laura sarà funzione della possibilità di giustificare il ritratto e preservarlo da ogni accusa di discendere da una peccaminosa e pervertita cupiditas videndi. (Verba videndi: «vedrian», «la vide», «occhi suoi»). E ciò proprio in quanto la cupiditas è sublimata e col-

47. Gianfranco Contini, Petrarca e le arti, cit., p. 117. Roberto LongHI, Proposte per una critica d'arte, "Paragone», I, 1950, ora in Id., Critica d'arte e buon governo 1938-1969, Firenze, 1985, p. 11.

48. Maria Monica Donato, op. cit., p. 436.

49. Marco Santagata, ediz. cit., p. 401. 
locata nel luogo supremo, il paradiso delle forme pure e degli archetipi voluti da Dio. Il superamento della cupiditas determina la fondazione del ritratto. Non la sparizione dell'immagine ma il suo rilancio in un nuovo e più alto e nobile istituto.

La deduzione che Laura possa rappresentare l'immagine della bellezza non meno che la bellezza (quanto confessabile?)dell'immagine (ma non l'idea della bellezza in sé, andrà ancora precisato) sottostà alla condizione che questa bellezza risulti giustificabile dall'esperienza del processo di astrazione intellettuale che porta ad attingere alle idee pure create da Dio; o almeno con essa spiegabile. In fondo, come dicevamo, il processo è il medesimo che in "Movesi il vecchierel»; solo che in $R v f 77$ la realizzazione non è puramente mentale, ma finalizzata alla pratica figurativa e alla sua spiegazione per linee generali teoriche calate in un'ipotesi di descrizione progettuale ( Ma certo il mio Simon $\mathrm{fu}$ in paradiso...»), che ha insieme del paradossale, sul piano della verosimiglianza, ma del postulato su quello dell'assunzione di una precisa prospettiva filosofica.

Se l'icona ingaggiava immediatamente in una discussione teologica e fisica, sul piano cioè — per riassumerlo con una sinestesia- della possibilità di toccare il divino nell'immagine (di fatto le icone erano spesso sottoposte alla mano trepida o irosa dei fedeli che la consumavano), la questione ora è spostata sul piano gnoseologico e ontologico-estetico, a voler conferire terminologia filosofica a un discorso in versi che non vuole adottarla, pur mantenendosi sul tracciato con più coerenza e precisione di quel che ci si poteva attendere.

\section{Il committente}

Sull'altro versante, quello strettamente attinente all'arte liberale, assistiamo al progressivo scambio comunicativo e all'aggiustamento storico dei ruoli tra "produttore/creatore» e «fruitore». Con due portati epocali:

a) Poiché, innanzitutto, al creativismo platonico andrà pure fatto carico di riconoscere e assumere l'idea di auctor (riconosciuto dunque non solo per via analogica, cioè per comparazione con il Poeta) nella misura in cui si riconosce saldamente al facitore, all'artifex, l'ingenium. Ecco, precisamente: la detenzione dell'ars o ingenium: «Ingenium [...] que forma est anime unde opera hec que laudamus ac miramur velut e fonte procedunt» (A Guido Sette, Fam. V, 17, 8). ${ }^{50}$

Ovvero la concessione di un potere assimilabile a quello di un diodemiurgo che crea il mondo a partire dalle idee.

b) Se è vero che fa parte di una concezione moderna dell'arte liberale la discussione sull'arte medesima non più dall'esterno, ovvero sul piano teologico della liceità o meno dell'immagine, oppure tassonomico, con riguardo

50. Francesco Petrarca, Le familiari, edizione critica per cura di Vittorio Rossi, Firenze: Sansoni, II, p. 39. Cfr. Maria Monica DONATO, op. cit., p. 440. 
esclusivo alla tassonomia delle arti, ma dentro il processo di realizzazione del prodotto che non si configura più come mera reduplicazione di un modello tràdito, piuttosto come concezione e sforzo di «invenzione» personale del pittore-produttore in quanto artista, qui ci sono tutti gli ingredienti di una simile discussione, che annuncia, direbbe Belting, l'epoca dell'emancipazione dell'arte:

Nella mediazione estetica [del fenomeno dell'arte moderna rispetto all'icona] c'è un'altra possibilità dell'uso dell'immagine, su cui l'artista e l'osservatore s'intendono tra loro. Il soggetto assume il controllo dell'immagine e cerca di applicare nell'arte la sua comprensione metaforica del mondo. L'immagine, che d'ora in poi non solo sorge secondo le regole dell'arte ma diventa anche decifrabile in base ad esse, si offre alla riflessione dell'osservatore. Forma e contenuto cedono il loro senso immediato a quello mediato di un'esperienza estetica e di un argomento nascosto.

L'immagine è affidata all'osservatore. ${ }^{51}$

E la discussione tra i due prosegue, eccome. In 78.

\section{Rvf, 78}

Se «l'opera gentile» dell'ultimo sonetto ${ }^{52} s^{\prime}$ inscrive per definizione esplicita ancora nella "cortesia», il discorso piega per altra strada: qui si tratta della corrispondenza tra il soggetto dell'opera (Laura) e il suo ossessionato fruitore che ne vorrebbe ascoltare la voce dal quadro medesimo mentre «rispond[e] a' detti [suoi]", foss'anche per una sola volta di quelle mille ch'ebbe in grazia Pigmalione. Qui il desiderio di corrispondenza di amorosi sensi (davvero solo erotici come vorrebbero alcuni esegeti?) imprime alle definizione del ritratto un'accelerazione imprevista nella direzione, innanzitutto, dell'appercezione sensitiva. Non più la sola vista è coinvolta («alto concetto", "però che 'n vista si mostra», "aspetto", "l'immagine tua») ma anche — ed in opposizione- l'udito (la «voce», «ragionar co.llei», «ascolte», «risponder», "detti miei»), anche

51. Hans Belting, op. cit., p. 31.

52. Quando Simone (il «mio Simon» di 77) fu raggiunto dal nobile concepimento dell'idea che in mia vece (su mia committenza) gli mise in mano il bastoncino (verghetta o matita di piombo o stagno per disegnare), se alla sua opera nobile (il ritratto, il ritratto di una donna gentile per beltà) (Simone) avesse conferito insieme con i lineamenti anche voce e capacità d'intendere, mi avrebbe liberato il cuore di molte (ragioni di) sospiri, (sospiri) che mi fanno disprezzare ciò che (il ritratto? i desideri sessuali?) gli altri apprezzano: dal momento che la vista di lei (Laura) si mostra benevolmente modesta e i suoi tratti mi comunicano promesse di pace (ovvero di esaudimento).

Ma dopo che io prendo a dialogare e a ragionare d'amore con l'immagine di lei, pare che lei mi stia ad ascoltare con molta benevolenza, se non che non può rispondere alle parole che io pronuncio. O Pigmalione, quanto ti devi considerare soddisfatto della statua che scolpisti, se riuscisti a ottenere da lei mille volte ciò che io una sola (volta) desidererei (ossia "dimostrazioni d'amore», Leopardi o, visto il contesto, colloqui e ragionamenti orali d'amore, voce che esprime e dialoga d'amore). 
se voce e udito sono convocati in assenza, spietata dolorosa assenza comminata da un'impossibilità irrevocabile. Per cui i versi "però che 'n vista ella si mostra humile / promettendomi pace ne l'aspetto» ha il senso di un'allargata quanto sofferta sinestesia agognata quanto irrealizzabile. Possibile solo, eventualmente, per intervento di un miracolo divino, come per Pigmalione. Anche la possibile carica erotica (di cui infra) dal punto di vista della codificazione empirica del ritratto, avrà la funzione strumentale di esaltare il momento intensissimo del rapporto diretto con l'immagine nella sua concreta appartenenza al corpo vivo di Laura, al punto che la forza e la qualità del ritratto sono paradossalmente indicati da un'assenza, una deficienza.

Rosanna Bettarini: «5-6: m’avrebbe liberato da molti sospiri (cioè dal rimpianto che l'immagine non sia la donna vera) che mi fanno apparire di poco valore quella figura (ciò) che gli altri tengono per cara e preziosa. Vellutello, Gesualdo e in parte Tassoni riferiscono che...fan a "voce et intellecto", v. 4; altri interpreti malamente vedono in "ciò ch'altri à più caro" un'allusione ai desideri sensuali (cosa per altro contraddittoria perché non si vede chi sospiri per cosa che tiene a vile).» 53

E già nel commento a 77: «Con una specie di paradossale sdoppiamento mal compreso dagli esegeti (LXXVIII 5-6) il contemplante scrive che tanto più 'cara', rara e preziosa, è per gli altri la figura di Simone, quanto più a lui è vile, perché, secondo topoi collaudati, non "ha voce et intellecto" perché non ha vita e non risponde, come invece è viva e rispondente l'immagine eburnea di Pigmalione». 54

Ma davvero Petrarca può dire, comunque, anche per paradosso, che quella figura, quel ritratto realizzato dal suo Simone Martini, per lui è vile? Che lo disprezza? E chi sono gli altri che invece lo pregiano? Non era di esclusiva pertinenza del beneficato?

Nel quadro dei rapporti tra oralità e scrittura, tra vista e udito: «se gli avesse dato con la figura (i tratti visibili) anche la voce e la possibilità di comprendere, gli avrebbe liberato il petto di molti sospiri, cioè del rimpianto di non poter parlare, colloquiare con lei, il quale rimpianto (specifico: esclusivamente provocato dalla mancanza di reciprocità dialogica, ovvero desiderio di dialogare d'amore con lei) gli fa disprezzare quei desideri meramente sensuali e visivi (destati dalla cupiditas videndi) che gli altri invece apprezzano. Si tratta, vi voglio dire, di sospiri per l'assenza di mutua parola di ragionamenti d'amore, non di altro.

53. Francesco Petrarca, Canzoniere Rerum vulgarium fragmenta, cit., a cura di Rosanna Bettarini, p. 399.

54. Ivi, p. 395. SANTAGATA: «mi avrebbe alleggerito il cuore del peso di molti sospiri, di molta angoscia» (perché come spiega dopo, la donna ritratta, diversamente da quella reale, sembra umile e benigna); [...] Che... vile nel senso che i sospiri, la passione amorosa, gli fanno disprezzare ciò che più gli altri apprezzano. E' una formula ambigua, nella quale mi sembrano predominanti le connotazioni negative [...] irricevibile mi sembra la lettura: "i quali sospiri, suscitati da puri e nobili desideri, a me fanno considerare come vile quel che più gli altri appetiscono, cioè l'appagamento sensuale" (Chiari, ma anche Moschetti, Chiòrboli, Ponchiroli e Apollonio-Ferro). Perché p. vorrebbe liberarsi di nobili desideri?» p. 405. 


\section{Due sonetti nella storia dell'arte}

La forza di attrazione reciproca dei due sonetti in dittico non dipende, ovvio, solo dall'unicità dell'interlocutore, implicato in terza persona: e la terza persona rafforza la carica oggettiva delle affermazioni, mentre le riferisce a un più ampio uditorio, a un pubblico virtuale, senza perdere un'oncia del tono di commossa riconoscenza personale. Perché evidentemente anche il discorso teorico sulla pittura, - teorico: sempre nel senso che abbiamo premesso-, avanza intrecciato anche qui al vissuto esistenziale e, anzi, con un accresciuto tasso di investimento amoroso e angoscioso da parte di colui che si proclama sempre, ad ogni verso, come unico utente universale, unico coinvolto, oltre che nella lectio, nella passio.

Insomma, voglio anche dire che il sonetto coinvolge meno la classica discussione tassonomica sulle arti liberali (e la prevalenza della parola sull'immagine oppure la necessità che la fama degli artisti creatori di opere mute venga innalzata dalle opere parlanti degli scrittori, come tutti i commentatori spiegano nel reparto glossatorio $)^{55}$ che non il rapporto tra fruizione visiva e fruizione e godimento olistico dell'opera fatta da mano umana con mente «divina». Alla da più parti rilevata «freddezza» degli interventi petrarchiani sull'arte sparsi nelle opere corrisponde qui il calore di un'omaggio e un'apologetica appassionata.

La diatriba tassonomica con le sue consuete implicazioni gerarchiche è superata anche perché qui non si tratta di una linea con due poli opposti (parola-immagine, scrittura-ritratto) ma di un triangolo ai cui vertici s'inscrivono oralità, scrittura e pittura, secondo la nuova svolta epistemologica, — già saggiata da molti studiosi- che ormai sancisce la divaricazione antropologica tra ciò che per praticità condensiamo nei termini di «scritto» e "orale». E tutto ciò nel quadro di un Canzoniere dove la donna, prevalentemente muta, coincide per sineddoche dominante quasi esclusivamente con il suo sguardo e i suoi occhi. Se gli occhi separano e intellettualizzano, astraggono il rapporto con l'oggetto, la parola orale rinvia al contatto fisico del corpo attraverso la voce, che prima di essere eventualmente pregna di parole «benigne», a livello di significato installa un rapporto concreto-sensibile tra i due soggetti.

Con ciò Petrarca, calandola nel pieno dell'esperienza personale, esperienza di amante che non smette di riflettere sulla fenomenologia del proprio amore, ed esperienza di poeta che ritrae e discute in versi un ritratto inventando, non il «ritratto di un dipinto» come vuole Pommier, quanto il ritratto del soggetto coinvolto nell'appercezione interessata di un ritratto dipinto.

Se in 77 il ritratto di Simone era spiegato e descritto attraverso l'itinerario esperienziale dell'artifex, che per ben operare sale in paradiso, in 78 il medesi-

55. «In Petrarca la scrittura non vuole chiose, diversioni, pause illustrative [...]per converso, a più riprese fa intendere che l'immagine, per significare, vuole un supporto verbale» (Monica 437. Nel 1353, com'è noto, a Milano, di fronte al ritratto di Ambrogio posto accanto al suo sepolcro, p. dirà: "Manca solo la voce, perché creda di vedere Ambrogio vivo» (Fam., XVI, 11) Cfr. Maurizio BeTTINI, Francesco Petrarca sulle arti figurative, cit., p. 222-223. 
mo ritratto è spiegato e descritto attraverso la reazione emotiva del lector o spectator. Quasi una catastrofe per eccesso di efficacia.

In grazia della forza incalzante del desiderio - emarginato, invece, in tutti gli altri luoghi ove si accenna alle arti liberali- lo stereotipo del ritratto cui manca la parola e l'antica gerarchia che vuole l'imago sottomessa al verbum, vengono rovesciati. L'opera è nobile. Un Poeta esalta un pittore. L'assenza di voce è la misura della forza inaudita di coinvolgimento totale del Ritratto, teste il Committente come protagonista assoluto di un'esperienza da laboratorio.

Presi assieme in binomio, 77 e 78 rinviano allora ai due tratti essenziali del genere figurativo moderno. Uno, diciamo così, oggettivo, per cui il ritratto è insieme ritratto ideale — che s'informa cioè a un'idea archetipica astratta- e ritratto fisico (non ancora fisiognomico) immediatamente riconoscibile nel modello umano. Che è il dualismo su cui s'incamminerà la storia del ritratto, coincidenza tra carne visibile e riconoscibile e eidon; e che avrà nel Rinascimento il suo momento d'equilibrio irripetibile e in Panowski il suo maggior teorico. ${ }^{56}$ E l'altro a parte subjecti, ossia il senso di mancanza infinita e di distanza incommensurabile, di irraggiungibiltà, — una volta abbandonata l'esperienza del contatto diretto con il divino attraverso l'icona che il pellegrino appercepisce per tutti i pori del suo corpo ben macerato-, in cui l'arte, una volta fondatasi sulle basi della ragione ottica e del dominio scopico dell'ideale e del reale, —umano e naturale_-, getta la soggettività moderna. 